\title{
A Mollification Regularization Method for a Fractional-Diffusion Inverse Heat Conduction Problem
}

\author{
Zhi-Liang Deng, ${ }^{1}$ Xiao-Mei Yang, ${ }^{2}$ and Xiao-Li Feng ${ }^{3}$ \\ ${ }^{1}$ School of Mathematical Sciences, University of Electronic Science and Technology of China, \\ Chengdu 610054, China \\ ${ }^{2}$ School of Mathematics, Southwest Jiaotong University, Chengdu 610031, China \\ ${ }^{3}$ Department of Mathematical Sciences, Xidian University, Xi'an 710071, China
}

Correspondence should be addressed to Zhi-Liang Deng; zhiliangdeng@gmail.com

Received 12 June 2012; Revised 19 December 2012; Accepted 20 December 2012

Academic Editor: Fatih Yaman

Copyright (C) 2013 Zhi-Liang Deng et al. This is an open access article distributed under the Creative Commons Attribution License, which permits unrestricted use, distribution, and reproduction in any medium, provided the original work is properly cited.

\begin{abstract}
The ill-posed problem of attempting to recover the temperature functions from one measured transient data temperature at some interior point of a one-dimensional semi-infinite conductor when the governing linear diffusion equation is of fractional type is discussed. A simple regularization method based on Dirichlet kernel mollification techniques is introduced. We also propose $a$ priori and a posteriori parameter choice rules and get the corresponding error estimate between the exact solution and its regularized approximation. Moreover, a numerical example is provided to verify our theoretical results.
\end{abstract}

\section{Introduction}

It is well known that the classical diffusion equation requires second spatial derivatives and first time derivatives. However, people are shifting their partial focus to fractional-order differential equations with the realization that the use of fractional-order derivatives and integrals leads to formulas of certain physical processes which is more economical and useful than the classical approach in terms of Fick's laws of diffusion [1-4]. Some time fractional diffusion equations involving only a first-order spatial derivative and a halforder time derivative are successfully used for modeling some anomalous diffusion physical phenomena (see, e.g., [5-7] and the references therein). One can also find some applications of such fractional diffusion equations in several scenarios in $[2,7]$, such as relaxation to equilibrium in systems (such as polymers chains and membranes) with long temporal memory, anomalous transport in disordered systems, diffusion on fractals, and to model non-Markovian dynamical processes in protein folding. Fractional diffusion equations have been extensively investigated both in theory itself and numerical computation based on the broad applications in many application fields, especially in describing phenomena related to anomalous diffusion processes. The following is a partial list of articles which contain theoretical results and numerical tests. Some fundamental solutions and Green functions of fractional differential equations are given in $[8,9]$. Finite difference techniques [10-12] and finite element methods $[13,14]$ provide some efficiency numerical results for several kinds of fractional differential equations. In [15], the decomposition method is used to construct analytical approximate solutions of time-fractional wave equation subject to specified boundary conditions. An analytical solution of a fractional diffusion equation by Adomian decomposition method is presented in [16]. One can also refer [17-19] to the regularity of the solution of fractional diffusion equations and some a priori estimates.

In this paper, we consider the following one-dimensional fractional diffusion problem on a semi-infinite slab: suppose the temperature $f(t)$ at some interior point $x=x_{0}>0$ is approximately measurable. For convenience, we set $x_{0}=1$. The temperature $u(x, t)$ at the $0<x<1$ is desired and 


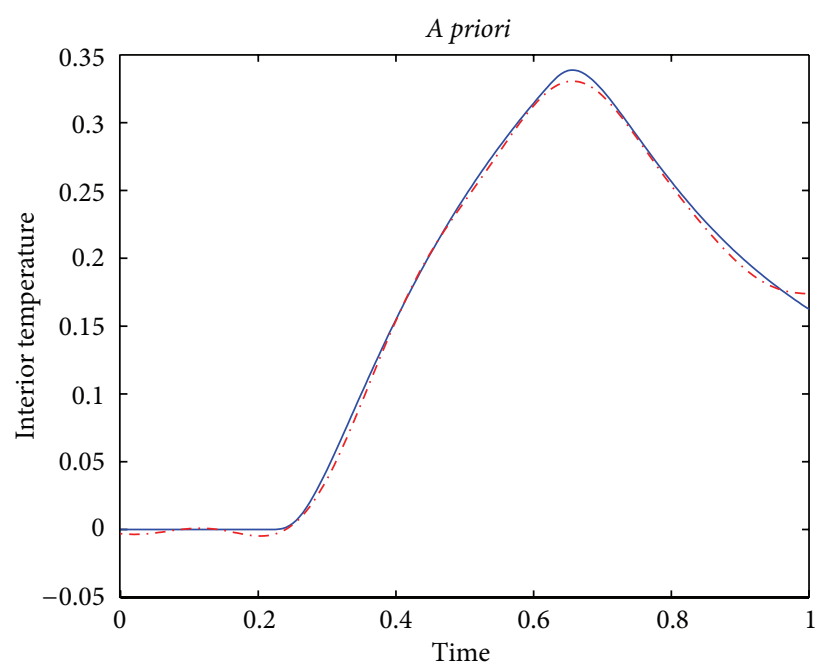

(a)

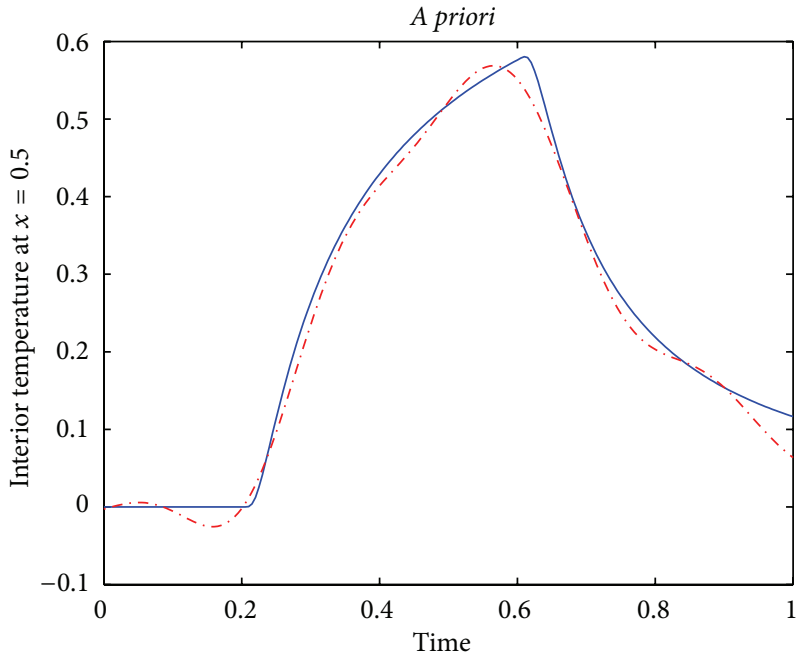

(b)

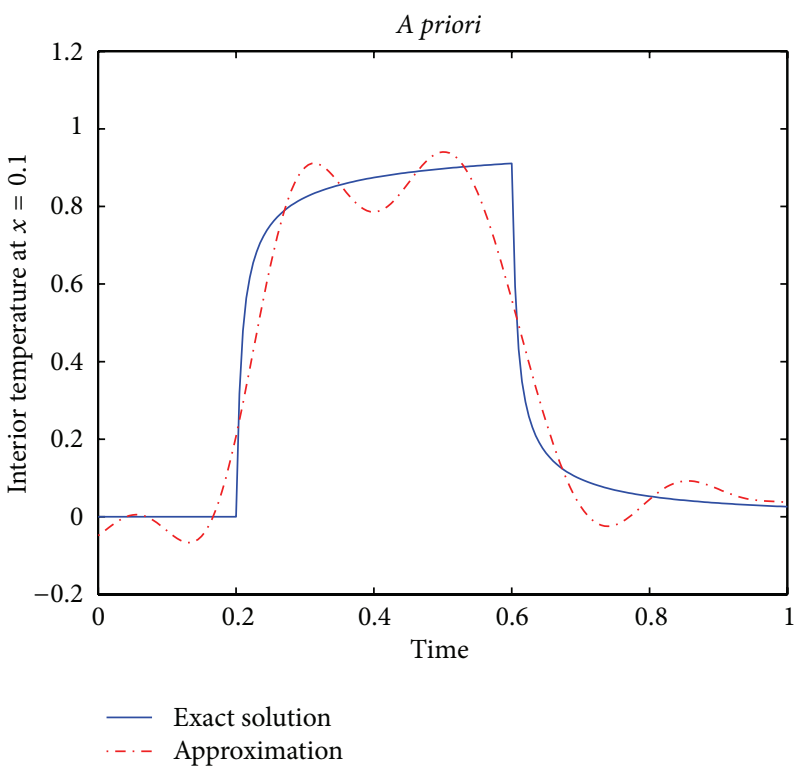

(c)

FIGURE 1: The comparisons of exact solution and regularization solution at (a) $x=0.9$, (b) $x=0.5$, (c) $x=0.1, v=34.3956, \delta=0.01$.

unknown. The mathematical description of the fractional diffusion problem is listed in the following. The unknown temperature $u(x, t)$ satisfies

$$
\begin{gathered}
\frac{\partial}{\partial x} u(x, t)=-\frac{1}{\sqrt{\kappa}} \frac{\partial^{1 / 2}}{\partial t^{1 / 2}} u(x, t)+\frac{u_{0}}{\sqrt{\pi \kappa t}}, \quad x>0, t \geq 0, \\
u(1, t)=f(t),
\end{gathered}
$$

where $\kappa$ is the constant diffusivity coefficient, $u_{0}=u(x, 0)$, a constant. The half-time differentiation $\left(\partial^{1 / 2} / \partial t^{1 / 2}\right) u(x, t)$ indicates the Riemann-Liouville fractional derivative with order $\alpha=1 / 2$ which is given by the convolution integral

$$
\begin{aligned}
& \left(\partial^{\alpha} u\right)(t) \\
& =\frac{1}{\Gamma(1-\alpha)} \frac{d}{d t} \int_{0}^{t} u(s)(t-s)^{-\alpha} d s, \quad 0 \leq t \leq T, 0<\alpha<1,
\end{aligned}
$$

where $\Gamma(\cdot)$ is the Gamma function. More detailed information on fractional derivatives and a general historical perspective may be found in $[7,20,21]$. The situations we will treat are those in which the system is initially at equilibrium, so that

$$
u(x, t)=u_{0}, \quad t<0, x \geq 0 .
$$




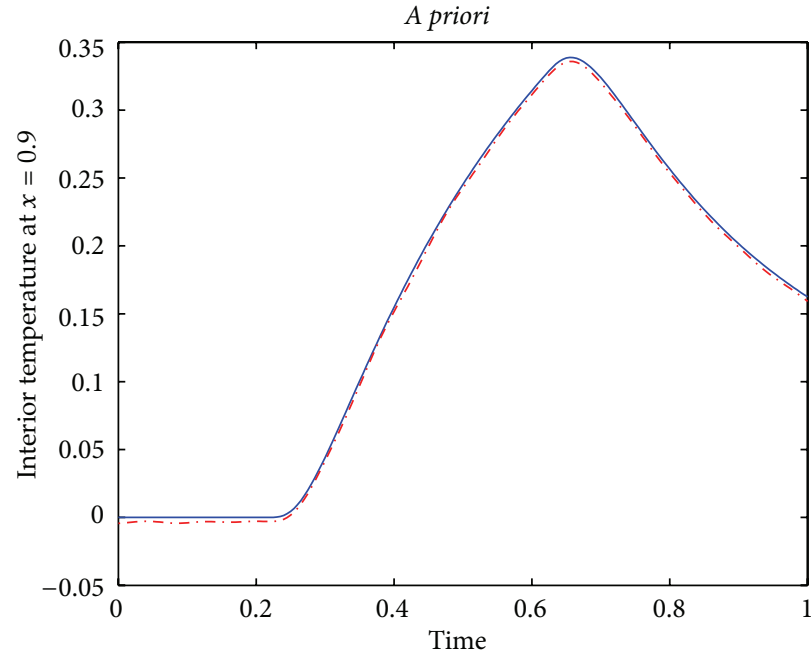

(a)

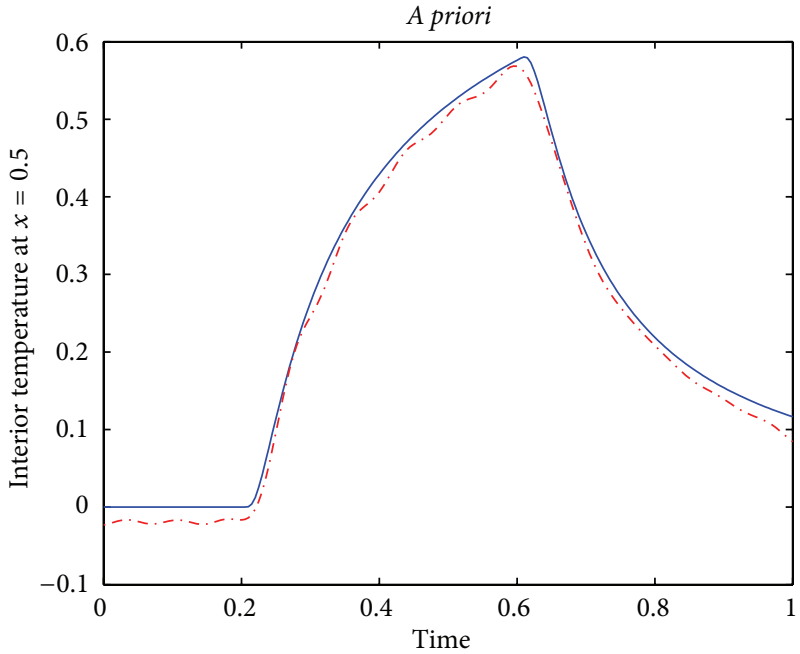

(b)

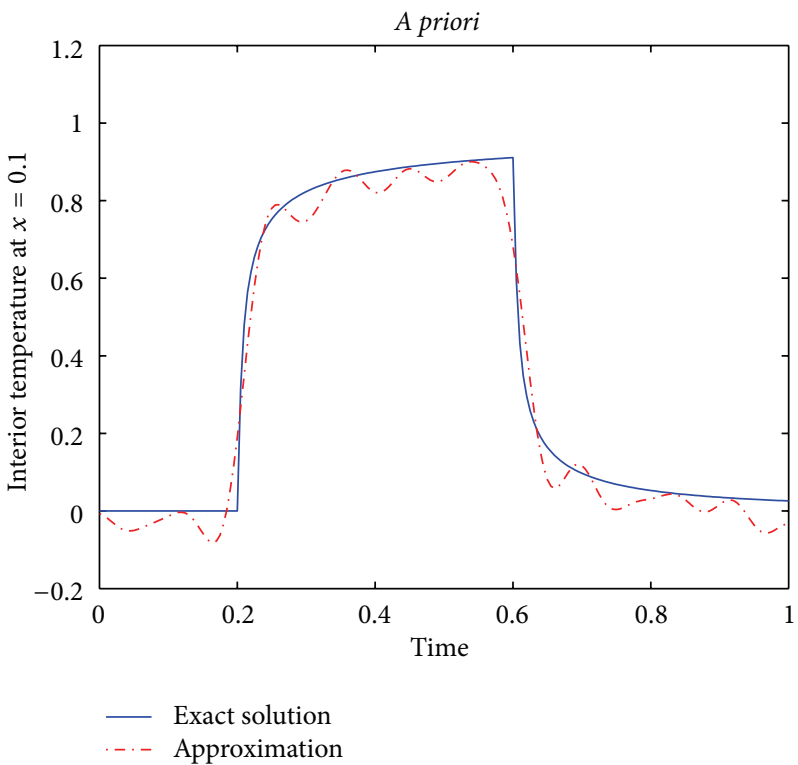

(c)

FIgURE 2: The comparisons of exact solution and regularization solution at (a) $x=0.9$, (b) $x=0.5$, (c) $x=0.1, v=83.1949, \delta=0.001$.

At $t=0$ a perturbation of the system commences by some unspecified process occurring at the boundary. During times of interest $T$, this perturbation does not affect regions remote from the $x=0$ boundary, so that the relationship

$$
\lim _{x \rightarrow+\infty} u(x, t)=u_{0}, \quad t \leq T
$$

applies. In [22], Murio discussed the similar problems using mollification method with Gauss kernel. The idea used in this current work is a development of the ideas in [22]. However, there is only formal stability and discrete error discussion in the cited paper. Here, we give some analysis of error estimates under a priori and a posteriori regularization parameter, and a comparison of those two choice methods in our regularization method. In the following, the regularization method is outlined.
In order to simplify the Fourier analysis of fractional diffusion problem (1), and in the rest of the paper, we assume without loss of generality, $\kappa=1, u_{0}=0$. We also assume that all the functions involved are $L^{2}(\mathbb{R})$ and use the corresponding $L^{2}$ norm, as defined in the following, to measure errors:

$$
\|y\|=\left(\int_{\mathbb{R}}|y(t)|^{2} d t\right)^{1 / 2}
$$

If the Fourier transform of a function $y(t)$ is written as

$$
\widehat{y}(\xi)=\frac{1}{\sqrt{2 \pi}} \int_{-\infty}^{+\infty} y(t) e^{-i \xi t} d t, \quad i=\sqrt{-1},
$$




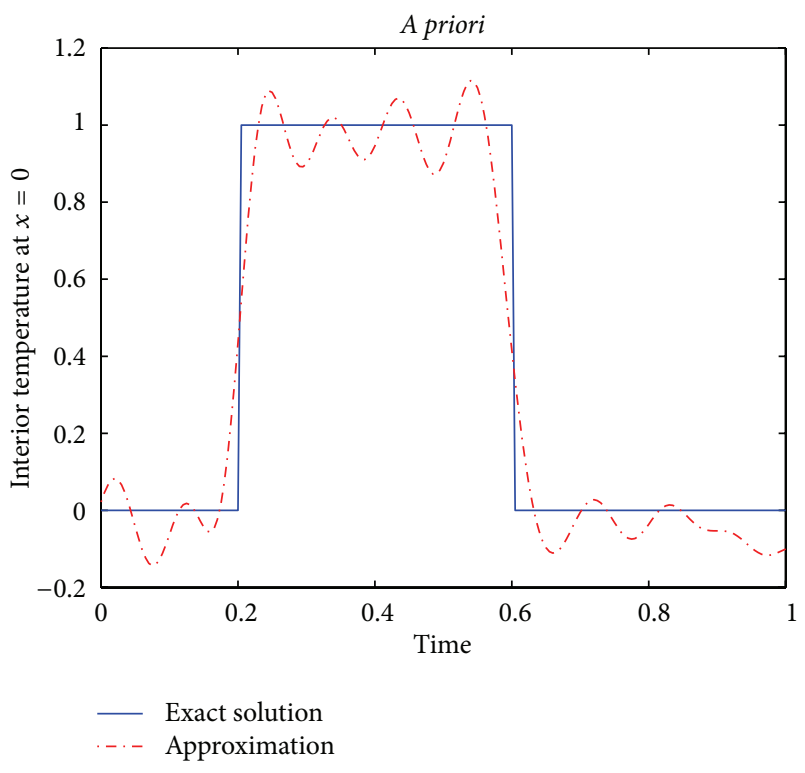

FIGURE 3: The comparisons of exact solution and regularization solution at $x=0, v=83.1949, \delta=0.001$.

then the Fourier inversion formula reads

$$
y(t)=\frac{1}{\sqrt{2 \pi}} \int_{-\infty}^{+\infty} \widehat{y}(\xi) e^{i \xi t} d \xi .
$$

Fourier transforming (1) with respect to variable $t$, it follows that

$$
\frac{\partial}{\partial x} \widehat{u}(x, \xi)=-\sqrt{i \xi} \widehat{u}(x, \xi), \quad x>0, \xi \in \mathbb{R}
$$

according to the fact that [7]

$$
\frac{\partial^{1 / 2}}{\partial t^{1 / 2}} h(t)=\sqrt{i \xi} \widehat{h}(\xi)
$$

The first-order ordinary differential equation (8) has the general solution

$$
\widehat{u}(x, \xi)=\widehat{u}(1, \xi) e^{\sqrt{i \xi}(1-x)}=\widehat{f}(\xi) e^{\sqrt{i \xi}(1-x)},
$$

or equivalently,

$$
e^{-\sqrt{i \xi}(1-x)} \widehat{u}(x, \xi)=\widehat{f}(\xi) .
$$

Now, we can write the problem (1) as

$$
\widehat{A}(\xi, x) \widehat{u}(x, \xi)=\widehat{f}(\xi),
$$

where $\widehat{A}(\xi, x):=e^{-\sqrt{i \xi}(1-x)}$ is a multiplication operator.

Since we require $\widehat{u}(x, \cdot) \in L^{2}(\mathbb{R})$ and $u_{0}=0$, we see from (4) and (10) that the real part of $\sqrt{i \xi}$ is positive. Thereby, the multiplication operator $\widehat{A}(\xi, x)$ is a bounded linear operator for $0 \leq x<1$. Then its inverse operator is unbounded. Therefore problem (12) is linear ill posed. For the general theory of linear ill posed problems, we can refer to [23]. It is worth pointing out that ill posed problems of a large number of diffusion equations, both fractionalorder as well as integral order, have been discussed by many authors. Yang et al. [24-28] discuss the identification of source terms for some integral-order diffusion equations using some regularization strategies. Hon et al. $[29,30]$ apply some meshless methods to the ill posed problems of heat conduction equations. In [17, 22, 29, 31, 32], some uniqueness results and numerical methods are given for some fractional diffusion ill posed problems. Here, we apply a simple stabilizing method, namely, the mollification method with Dirichlet kernel [33], to stabilize the problem (1). Suppose that the measured data function $f_{\delta}(t)$ satisfies

$$
\left\|f-f_{\delta}\right\| \leq \delta
$$

where $\delta$ is noise level. Take the Dirichlet function

$$
D_{v}(t):=\sqrt{\frac{2}{\pi}} \frac{\sin v t}{t}
$$

as the mollifier kernel, where $v$ is a positive constant. Define operator $M_{v}$ as

$$
M_{\nu} u(x, t):=\int_{-\infty}^{+\infty} D_{v}(\tau) u(x, t-\tau) d \tau .
$$

Then, we have the following associated problem: for some $\delta>$ 0 , find $M_{v} u_{\delta}(x, t)$ that satisfies

$$
\begin{gathered}
\frac{\partial}{\partial x} M_{\nu} u_{\delta}(x, t)=-\frac{\partial^{1 / 2}}{\partial t^{1 / 2}} M_{v} u_{\delta}(x, t), \quad x>0, t \geq 0, \\
M_{\nu} u_{\delta}(1, t)=M_{v} f_{\delta}(t) .
\end{gathered}
$$

In fact, $v$ plays the role of regularization parameter.

In Section 2 an a priori parameter choice rule and the corresponding error estimate are discussed. In Section 3, we propose an $a$ posteriori parameter choice rule and get the error bound. Numerical tests are given in Section 4.

\section{The Error Estimate with a Priori Parameter Choice}

In this section, the error estimate of the mollification regularization method will be derived under the a priori parameter choice rule. Suppose that the following source condition holds:

$$
\|u(0, \cdot)\| \leq E
$$

The source condition (17) is indispensable, otherwise, there can be no uniform convergence rates for any regularization method, that is, the convergence is arbitrarily slow 


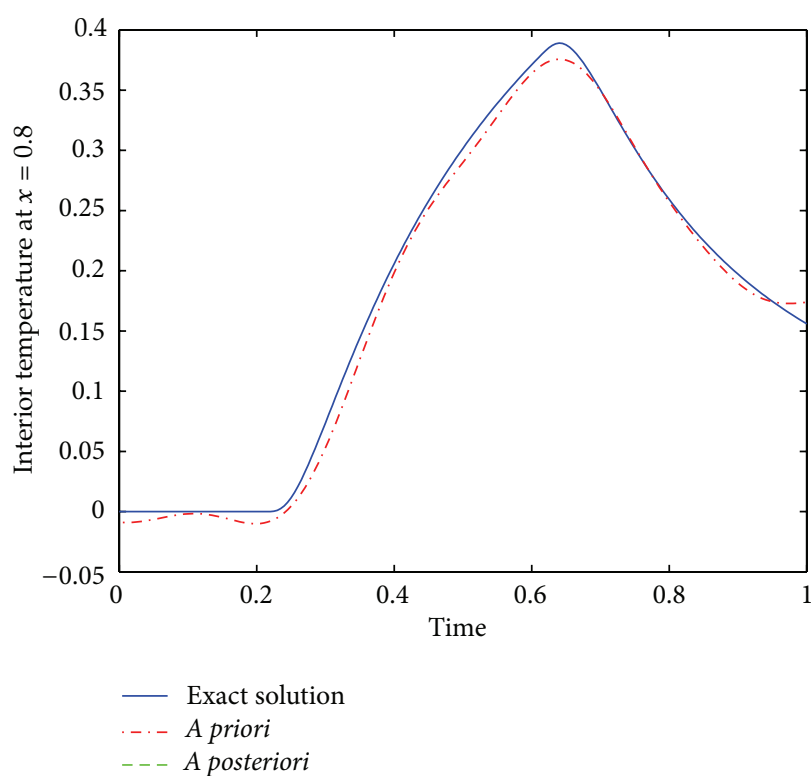

(a)

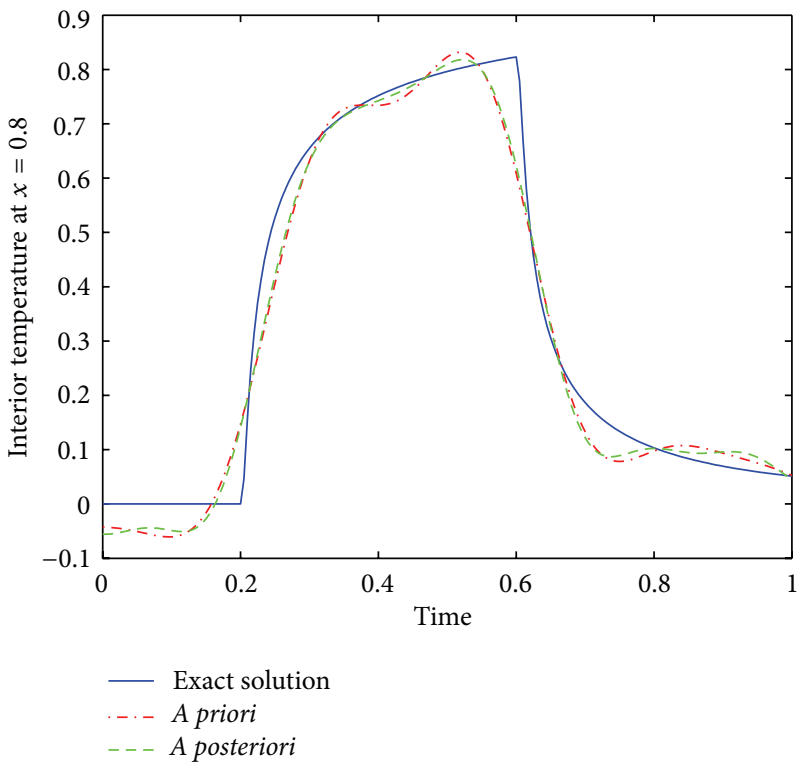

(b)

FIGURE 4: The comparisons of exact solution and regularization solution at (a) $x=0.8$, (b) $x=0.2$ with $\delta=0.01$.

(see Proposition 3.11 in [23]). For $0<x<1$, by Parseval formula and triangle inequality, we know that

$$
\begin{aligned}
& \left\|M_{v} u_{\delta}(x, \cdot)-u(x, \cdot)\right\| \\
& =\left\|\widehat{M_{v} u_{\delta}}(x, \cdot)-\widehat{u}(x, \cdot)\right\|
\end{aligned}
$$

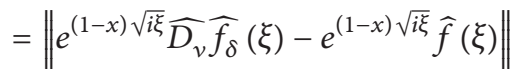

$$
\begin{aligned}
& \leq\left\|e^{(1-x) \sqrt{i \xi}} \widehat{D_{v}} \widehat{f_{\delta}}(\xi)-e^{(1-x) \sqrt{i \xi}} \widehat{D_{v}} \widehat{f}(\xi)\right\| \\
& +\left\|e^{(1-x) \sqrt{i \xi}} \widehat{D_{v}} \widehat{f}(\xi)-e^{(1-x) \sqrt{i \xi}} \widehat{f}(\xi)\right\| \\
& :=I_{1}+I_{2} \text {. }
\end{aligned}
$$

Since $\left|e^{\sqrt{i \xi}}\right|=e^{\sqrt{|\xi| / 2}}$, for $I_{1}$, we get by (13)

$$
\begin{aligned}
I_{1} & =\left\|e^{(1-x) \sqrt{i \xi}} \widehat{D_{v}} \widehat{f_{\delta}}(\xi)-e^{(1-x) \sqrt{i \xi}} \widehat{D_{v}} \widehat{f}(\xi)\right\| \\
& =\left(\int_{-v}^{v}\left|e^{(1-x) \sqrt{i \xi}}\left(\widehat{f_{\delta}}-\widehat{f}\right)\right|^{2} d \xi\right)^{1 / 2} \\
& \leq e^{(1-x) \sqrt{v / 2}} \delta .
\end{aligned}
$$

For $I_{2}$, we use the source condition (17) and obtain

$$
\begin{aligned}
& I_{2}=\left\|e^{(1-x) \sqrt{i \xi}} \widehat{D_{v}} \widehat{f}(\xi)-e^{(1-x) \sqrt{i \xi}} \widehat{f}(\xi)\right\| \\
& =\left(\int_{|\xi| \geq v}\left|e^{(1-x) \sqrt{i \xi}} \widehat{f}(\xi)\right|^{2} d \xi\right)^{1 / 2} \\
& =\left(\int_{|\xi| \geq v}\left|e^{(1-x) \sqrt{i \xi}} e^{-\sqrt{i \xi}} \widehat{u}(0, \xi)\right|^{2} d \xi\right)^{1 / 2} \\
& \leq e^{-\sqrt{v / 2} x} E \text {. }
\end{aligned}
$$

Therefore,

$$
\left\|M_{\nu} u_{\delta}(x, \cdot)-u(x, \cdot)\right\| \leq e^{(1-x) \sqrt{v / 2}} \delta+e^{-\sqrt{v / 2} x} E .
$$

Minimizing the right-hand side of (21), we set $e^{(1-x) \sqrt{v / 2}} \delta=$ $e^{-\sqrt{v / 2} x} E$ and get

$$
v=2\left(\log \frac{E}{\delta}\right)^{2}
$$

which also implies

$$
\left\|M_{\nu} u_{\delta}(x, \cdot)-u(x, \cdot)\right\| \leq 2 E^{1-x} \delta^{x}, \quad \text { for } 0<x<1 .
$$

Therefore, we get the following theorem.

Theorem 1. Assume conditions (13), (17) hold. If the regularization parameter $v$ is taken by (22), then for $0<x<1$, there holds the error estimate (23).

Remark 2. The error estimate (23) is order optimal in the sense of Tautenhahn [34]. In our application $\|u(0, \cdot)\|$ is 
usually not known, therefore we have no exact a priori bound $E$ and cannot choose the parameter $v$ according to (22). However, if selecting $v=2(\log (1 / \delta))^{2}$, we can obtain the convergence rate

$$
\left\|M_{v} u_{\delta}(x, \cdot)-u(x, \cdot)\right\| \leq 2 \delta^{x}, \quad \text { for } 0<x<1 .
$$

Theorem 1 provides no information about the convergence and convergence rates of $M_{v} u_{\delta}(x, \cdot)$ at $x=0$. The question is settled by our next result. We now give the error estimate at $x=0$ under a stronger $a$ priori assumption

$$
\|u(0, \cdot)\|_{p} \leq E, \quad p>0
$$

where $\|\cdot\|_{p}$ denotes the norm on Sobolev space $H_{p}(\mathbb{R})$ defined by

$$
\|f\|_{p}^{2}:=\int_{-\infty}^{\infty}\left(1+\xi^{2}\right)^{p}|\widehat{f}(\xi)|^{2} d \xi
$$

for $f \in H^{p}(\mathbb{R})$. We only need to reestimate $I_{2}$ for $x=0$. Under the stronger a priori bound (25), it is not hard to get

$$
\begin{aligned}
I_{2} & =\left(\int_{|\xi| \geq v}|\widehat{u}(0, \xi)|^{2} d \xi\right)^{1 / 2} \\
& =\left(\int_{|\xi| \geq v}\left(1+\xi^{2}\right)^{-p}\left(1+\xi^{2}\right)^{p}|\widehat{u}(0, \xi)|^{2} d \xi\right)^{1 / 2} \\
& \leq \frac{1}{\left(1+v^{2}\right)^{p}} E \leq \frac{E}{v^{2 p}} .
\end{aligned}
$$

Then, (18), (19), and (27) lead to the following error bound:

$$
\left\|M_{\nu} u_{\delta}(0, \cdot)-u(0, \cdot)\right\| \leq e^{\sqrt{v / 2}} \delta+\frac{E}{v^{2 p}}
$$

The error bound (28) does not provide the convergence as $\delta \rightarrow 0$ obviously. Hence, we need to choose a proper parameter $v$. For this reason, some proper lower bound of the right-hand side of (28) should be given. In order to minimize the right-hand side of (28), it is necessary to introduce the following lemma and its proof can be found in [34].

Lemma 3 (see [34]). Let the function $f(\lambda):(0, a] \rightarrow \mathbb{R}$ be given by

$$
f(\lambda)=\lambda^{b}\left[d \log \frac{1}{\lambda}\right]^{-c}
$$

with a constant $c \in \mathbb{R}$ and positive constants $a<1, b$ and $d$, then for the inverse function $f^{-1}(\lambda)$ one has

$$
f^{-1}(\lambda)=\lambda^{1 / b}\left[\frac{d}{b} \log \frac{1}{\lambda}\right]^{c / b}(1+o(1)) \quad \text { for } \lambda \longrightarrow 0 .
$$

Minimizing the right-hand side of (28), we let $e^{\sqrt{v / 2}} \delta=$ $E / \nu^{2 p}$. Denote $e^{-\sqrt{v / 2}}:=\lambda$. Simple computation shows $\lambda(\log (1 / \lambda))^{-4 p}=\delta 2^{2 p} / E$. By using (30) in Lemma 3, we obtain

$$
\lambda=\frac{\delta 2^{2 p}}{E}\left(\log \frac{E}{\delta 2^{2 p}}\right)^{4 p}(1+o(1)), \quad \text { for } \delta \longrightarrow 0,
$$

which also implies that

$$
v=2 \log ^{2}\left(\frac{\delta 2^{2 p}}{E}\left(\log \frac{E}{\delta 2^{2 p}}\right)^{4 p}\right)(1+o(1)), \quad \text { for } \delta \longrightarrow 0 .
$$

Therefore, the following estimate holds

$$
\begin{aligned}
& \left\|M_{v} u_{\delta}(0, \cdot)-u(0, \cdot)\right\| \\
& \quad \leq C E\left(2 \log ^{2}\left(\frac{\delta 2^{2 p}}{E}\left(\log \frac{E}{\delta 2^{2 p}}\right)^{4 p}\right)\right)^{(-2 p)},
\end{aligned}
$$

where $C$ is a constant, which also shows that the convergence rate at $x=0$ is logarithmic.

Theorem 4. Assume conditions (13), (25) hold. If the regularization parameter $v$ is taken as given by (32), then the error estimate (33) holds.

Remark 5. From the error estimate (23), as $x \rightarrow 0$, we see that the accuracy of regularization solution becomes progressively lower and even cannot get convergence for $x=$ 0 . This is common in the theory of ill posed problems. Nevertheless, if a stronger a priori assumption (25) is imposed, the regularization solution converges to the exact solution at $x=0$, but only in a slower way.

\section{The Error Estimate with a Posteriori Parameter Choice}

In this section, we consider the a posteriori regularization parameter choice rule. Choose the regularization parameter $v$ as the solution of the equation

$$
d(v):=\left\|\widehat{D_{v}} \widehat{f_{\delta}}(\cdot)-\widehat{f_{\delta}}(\cdot)\right\|=\tau \delta,
$$

where $\tau>1$ is a constant. To establish existence and uniqueness of solution of (34), we need the following lemma.

Lemma 6. If $\delta>0$, then there hold the following:

(a) $d(v)$ is a continuous function;

(b) $\lim _{v \rightarrow 0} d(v)=\left\|\widehat{f_{\delta}}\right\|$;

(c) $\lim _{v \rightarrow+\infty} d(v)=0$;

(d) $d(v)$ is a strictly decreasing function.

The proof is very easy and we omit it here. Denote

$$
\zeta(x, t):=M_{v} u_{\delta}(x, t)-u(x, t) .
$$

We give the main result of this section as follows.

Theorem 7. Assume the conditions (11) and (17) hold and $\tau>$ 1. Take the solution $v$ of (34) as the regularization parameter, then there holds the error estimate for $0<x<1$ :

$$
\left\|M_{\nu} u_{\delta}(x, t)-u(x, t)\right\| \leq C(E, \tau) E^{1-x} \delta^{x},
$$

where $C(E, \tau)$ is a constant depending on $E, \tau$. 
TABLE 1: Errors with a priori rule.

\begin{tabular}{ccccr}
\hline$x$ & & & \multicolumn{2}{c}{$\delta=0.001$} \\
\\
\hline 0.1 & $e_{r}$ & $e_{i}$ & $e_{r}$ & 0.7643 \\
0.5 & 0.1590 & 1.2154 & 0.1000 & 0.2370 \\
0.9 & 0.0596 & 0.2729 & 0.0517 & 0.0443 \\
\hline
\end{tabular}

Proof. By (35), the Parseval formula, triangle inequality, and (17), we have

$$
\begin{aligned}
\|\zeta(0, \cdot)\| & =\left\|M_{\nu} u_{\delta}(0, \cdot)-u(0, \cdot)\right\|=\left\|\widehat{M_{\nu} u_{\delta}}(0, \cdot)-\widehat{u}(0, \cdot)\right\| \\
& =\left\|\widehat{D_{v}} \widehat{u_{\delta}}(0, \cdot)-\widehat{u}(0, \cdot)\right\|=\left\|\widehat{D_{v}} e^{\sqrt{i \xi}} \widehat{f_{\delta}}-e^{\sqrt{i \xi}} \widehat{f}\right\| \\
& =\left\|\widehat{D_{v}} e^{\sqrt{i \xi}}\left(\widehat{f_{\delta}}-\widehat{f}\right)+\left(\widehat{D_{v}}-1\right) e^{\sqrt{i \xi}} \widehat{f}\right\| \\
& \leq\left\|\widehat{D_{v}} e^{\sqrt{i \xi}}\left(\widehat{f_{\delta}}-\widehat{f}\right)\right\|+E \\
& \leq e^{\sqrt{v / 2}} \delta+E .
\end{aligned}
$$

By virtue of (34), we know that

$$
\begin{aligned}
\tau \delta & =\left\|\widehat{D_{v}} \widehat{f_{\delta}}-\widehat{f_{\delta}}\right\|=\left\|\left(\widehat{D_{v}}-1\right)\left(\widehat{f_{\delta}}-\widehat{f}+\widehat{f}\right)\right\| \\
& \leq\left\|\left(\widehat{D_{v}}-1\right)\left(\widehat{f_{\delta}}-\widehat{f}\right)\right\|+\left\|\left(\widehat{D_{v}}-1\right) \widehat{f}\right\| \\
& \leq \delta+\left\|\left(\widehat{D_{v}}-1\right) e^{-\sqrt{i \xi}} \widehat{u}(0, \xi)\right\| \\
& \leq \delta+e^{-\sqrt{v / 2}} E,
\end{aligned}
$$

which means

$$
(\tau-1) \delta \leq e^{-\sqrt{v / 2}} E .
$$

Thus it is obvious that

$$
e^{\sqrt{v / 2}} \delta \leq \frac{E}{\tau-1}
$$

Moreover, inserting (40) into (37), we get the following inequality:

$$
\|\zeta(0, \cdot)\| \leq \frac{\tau}{\tau-1} E
$$

In addition,

$$
\begin{aligned}
\|\zeta(1, \cdot)\| & =\left\|M_{\nu} u_{\delta}(1, \cdot)-u(1, \cdot)\right\|=\left\|\widehat{M_{\nu} u_{\delta}}(1, \cdot)-\widehat{u}(1, \cdot)\right\| \\
& \leq\left\|\widehat{M_{\nu} u_{\delta}}(1, \cdot)-\widehat{u_{\delta}}(1, \cdot)\right\|+\left\|\widehat{u_{\delta}}(1, \cdot)-\widehat{u}(1, \cdot)\right\| \\
& \leq \tau \delta+\delta=(\tau+1) \delta .
\end{aligned}
$$

It is easy to see

$$
\begin{aligned}
\|\zeta(x, \cdot)\|^{2} \\
\quad=\int_{-\infty}^{+\infty}\left|\widehat{D_{v}} \widehat{u_{\delta}}(x, \xi)-\widehat{u}(x, \xi)\right|^{2} d \xi \\
=\int_{-\infty}^{+\infty}\left|\widehat{D_{v}} e^{\sqrt{i \xi(}(1-x)} \widehat{f_{\delta}}(\xi)-e^{\sqrt{i \xi}(1-x)} \widehat{f}(\xi)\right|^{2} d \xi \\
=\int_{-\infty}^{+\infty}\left|e^{\sqrt{i \xi(1-x)}}\left(\widehat{D_{v}} \widehat{f_{\delta}}(\xi)-\widehat{f}(\xi)\right)\right|^{2} d \xi .
\end{aligned}
$$

Denote $\theta_{f}=: \widehat{D_{v}} \widehat{f_{\delta}}(\xi)-\widehat{f}(\xi)$. By using the Hölder inequality, we know that

$$
\begin{aligned}
& \|\zeta(x, \cdot)\|^{2} \\
& \quad=\int_{-\infty}^{+\infty}\left|e^{\sqrt{i \xi}(1-x)}\right|^{2}\left|\theta_{f}\right|^{2 x}\left|\theta_{f}\right|^{2(1-x)} d \xi \\
& \quad \leq\left[\int_{-\infty}^{+\infty}\left|\left(e^{\sqrt{i \xi}} \theta_{f}\right)^{2(1-x)}\right|^{1 /(1-x)} d \xi\right]^{1-x}\left[\int_{-\infty}^{+\infty}\left(\theta_{f}^{2 x}\right)^{1 / x} d \xi\right]^{x} \\
& \quad=\left[\int_{-\infty}^{+\infty}\left|\left(e^{\sqrt{i \xi}} \theta_{f}\right)^{2}\right| d \xi\right]^{1-x}\left[\int_{-\infty}^{+\infty}\left(\theta_{f}^{2}\right) d \xi\right]^{x} .
\end{aligned}
$$

Therefore, we obtain

$$
\|\zeta(x, \cdot)\| \leq\|\zeta(0, \cdot)\|^{1-x}\|\zeta(1, \cdot)\|^{x},
$$

which also implies that

$$
\begin{aligned}
\left\|M_{v} u_{\delta}(x, \cdot)-u(x, \cdot)\right\| & \leq\|\zeta(0, \cdot)\|^{1-x}\|\zeta(1, \cdot)\|^{x} \\
& \leq\left(\frac{\tau}{\tau-1} E\right)^{1-x}((\tau+1) \delta)^{x} .
\end{aligned}
$$

Therefore, we complete the proof.

\section{Numerical Examples}

For linear heat diffusion, analytic solutions for the temperature distribution $u(x, t), x>0, t>0$ in a semi-infinite solid with zero initial temperature, and $u(0, t):=H(t)$ at the surface, are obtained using the integral equation

$$
u(x, t)=\left(H * \frac{\partial}{\partial t} K\right)(x, t)=\int_{0}^{t} H(s) \frac{\partial}{\partial t} K(x, t-s) d s,
$$


TABLE 2: Errors with $a$ priori rule at $x=0$.

\begin{tabular}{ccc}
\hline$x$ & & $\delta=0.001$ \\
$e_{r}$ & 1.6748 \\
\hline 0
\end{tabular}

TABLE 3: The comparison between a priori and a posteriori rules with $\delta=0.01$.

\begin{tabular}{lllll}
\hline$x$ & \multicolumn{3}{c}{ A priori } & \multicolumn{2}{c}{ A posteriori } \\
\hline 0.8 & $e_{r}=0.0321$ & $e_{i}=0.1063$ & $e_{r}=0.0313$ & $e_{i}=0.1010$ \\
0.2 & $e_{r}=0.1175$ & $e_{i}=0.7817$ & $e_{r}=0.1085$ & $e_{i}=0.7214$ \\
\hline
\end{tabular}

where the kernel function

$$
K(x, t)=\operatorname{erfc}\left(\frac{x}{2 \sqrt{t}}\right)
$$

is the temperature distribution corresponding to a unit step boundary temperature, $H(t)=1, t>0$. Here, $\operatorname{erfc}(\cdot)$ denotes the complementary error function defined by

$$
\operatorname{erfc}(z):=\frac{2}{\sqrt{\pi}} \int_{z}^{\infty} e^{-t^{2}} d t
$$

We take the example from [22]. As an interesting and challenging test for the numerical method, we proposed the surface temperature function $H(t)$, which is 1 between 0.2 and 0.6 and zero otherwise. The exact solutions for the FICHP, in this example, are the functions

$$
u(x, t)= \begin{cases}0, & 0<t \leq 0.2, \\ \operatorname{erfc}\left(\frac{x}{2 \sqrt{t-0.2}}\right), & 0.2<t \leq 0.6, \\ \operatorname{erfc}\left(\frac{x}{2 \sqrt{t-0.2}}\right) & 0.6<t<\infty . \\ -\operatorname{erfc}\left(\frac{x}{2 \sqrt{t-0.6}}\right), & \end{cases}
$$

Consequently, the exact interior data temperature is given by

$$
f(t)= \begin{cases}0, & 0<t \leq 0.2, \\ \operatorname{erfc}\left(\frac{1}{2 \sqrt{t-0.2}}\right), & 0.2<t \leq 0.6 \\ \operatorname{erfc}\left(\frac{1}{2 \sqrt{t-0.2}}\right) & 0.6<t<\infty \\ -\operatorname{erfc}\left(\frac{1}{2 \sqrt{t-0.6}}\right), & \end{cases}
$$

Suppose the vector $F$ represents samples from the function $f(t)$. The noisy discrete data function is generated by adding random errors to the exact data function, at every grid point, that is,

$$
F_{\delta}(n)=F(n)+\epsilon_{n}, \quad\left|\epsilon_{n}\right| \leq \delta, \quad n=0,1, \ldots, N_{t},
$$

where the $\left(\epsilon_{n}\right)$ 's are independent random Gaussian variables with variance $\sigma^{2}=\epsilon^{2}$. The absolute and relative weighted $l^{2}$ errors for the recovered interior temperatures are calculated as

$$
\begin{gathered}
e_{i}:=\left[\frac{1}{N_{t}+1} \sum_{n=0}^{N_{t}}\left|M_{v} u_{\delta}(x, n)-u(x, n)\right|\right]^{1 / 2}, \\
e_{r}:=\frac{\left[\left(1 /\left(N_{t}+1\right)\right) \sum_{n=0}^{N_{t}}\left|M_{v} u_{\delta}(x, n)-u(x, n)\right|\right]^{1 / 2}}{\left[\left(1 /\left(N_{t}+1\right)\right) \sum_{n=0}^{N_{t}}|u(x, n)|^{2}\right]^{1 / 2}},
\end{gathered}
$$

respectively.

It is easy to implement the algorithm described in (16). Using an available fast Fourier transform (FFT) subroutine, a simple program was written to test the algorithm for the above example in Matlab. Firstly, some tests are implemented to verify the effectiveness of the regularization method under a priori parameter choice rule at several interior points. In addition, we would like to compare the a posteriori parameter choice rule (34) with the a priori parameter choice rule (22). The a priori bound $E$ in (22) can be calculated easily in this example. The Newton's bisection is used to solve (34), where we choose $\tau=1.1$.

Figures 1 and 2 provide the comparisons between the exact solutions and regularization solutions with error bound $\delta=0.01$ and $\delta=0.001$ at interior point $x=0.1,0.5,0.9$ using a priori parameter choice rule (22), respectively. Figure 3 gives the comparison between the exact solutions and regularization solutions with error bound $\delta=0.001$ at boundary point $x=0$ using a priori parameter choice rule (32). And intuitively, it seems that there are better numerical effects for closer distance from $x=1$. We list the error in Tables 1 and 2 to verify our result. Figure 4 demonstrates the comparisons between a priori and a posteriori parameter choice rules and Table 3 tells us that it has better effect using a posteriori parameter choice rule than a priori parameter choice rule. We also see that the difference of the numerical results between $a$ priori choice rule and a posteriori choice rule is slight, which agrees with our theoretical results.

\section{Acknowledgments}

The authors are indebted to the referees of this paper for their most helpful comments and suggestions, which helped to improve the presentation greatly. This work was supported by 
the Fundamental Research Funds for the Central Universities ZYGX2011J104 and SWJTU11BR078 and the NSF of China (Nos. 11126102, 11226040, and 11126187).

\section{References}

[1] V. V. Kulish and J. L. Lage, "Fractional-diffusion solution for transient local temperature and heat flux," Transactions of ASME, vol. 122, pp. 372-376, 2000.

[2] K. B. Oldham and J. Spanier, The Fractional Calculus: Theory and Application of Differential and Integration to Arbitrary Order, Academic Press, 1974.

[3] K. B. Oldham and J. Spanier, "A general solution of the diffusion equation for semiinfinite geometries," Journal of Mathematical Analysis and Applications, vol. 39, pp. 655-669, 1972.

[4] K. B. Oldham and J. Spanier, "The replacement of Fick's law by a formulation involving semidifferentiation," Journal of Electroanalytical Chemistry, vol. 26, pp. 331-341, 1970.

[5] R. Gorenflo, F. Mainardi, D. Moretti, and P. Paradisi, "Time fractional diffusion: a discrete random walk approach," Nonlinear Dynamics, vol. 29, no. 1-4, pp. 129-143, 2002.

[6] V. V. Anh and N. N. Leonenko, "Non-Gaussian scenarios for the heat equation with singular initial conditions," Stochastic Processes and their Applications, vol. 84, no. 1, pp. 91-114, 1999.

[7] I. Podlubny, Fractional Differential Equations, Academic Press, 1999.

[8] M. M. El-Borai, "The fundamental solutions for fractional evolution equations of parabolic type," Boletín de la Asociación Matemática Venezolana, vol. 6, no. 1, pp. 29-43, 2004.

[9] F. Mainardi, Y. Luchko, and G. Pagnini, "The fundamental solution of the space-time fractional diffusion equation," Fractional Calculus \& Applied Analysis, vol. 4, no. 2, pp. 153-192, 2001.

[10] F. Liu, V. Anhb, and I. Turnerb, "Numerical solution of the space fractional Fokker-Planck equation," Journal of Computational and Applied Mathematics, vol. 166, pp. 209-219, 2004.

[11] M. M. Meerschaert and C. Tadjeran, "Finite difference approximations for fractional advection-dispersion flow equations," Journal of Computational and Applied Mathematics, vol. 172, no. 1, pp. 65-77, 2004.

[12] S. B. Yuste, "Weighted average finite difference methods for fractional diffusion equations," Journal of Computational Physics, vol. 216, no. 1, pp. 264-274, 2006.

[13] G. J. Fix and J. P. Roop, "Least squares finite-element solution of a fractional order two-point boundary value problem," Computers \& Mathematics with Applications, vol. 48, no. 7-8, pp. 1017-1033, 2004.

[14] J. P. Roop, "Computational aspects of FEM approximation of fractional advection dispersion equations on bounded domains in $R^{2}$," Journal of Computational and Applied Mathematics, vol. 193, no. 1, pp. 243-268, 2006.

[15] Z. M. Odibat and S. Momani, "Approximate solutions for boundary value problems of time-fractional wave equation," Applied Mathematics and Computation, vol. 181, no. 1, pp. 767774, 2006.

[16] S. S. Ray and R. K. Bera, "Analytical solution of a fractional diffusion equation by Adomian decomposition method," Applied Mathematics and Computation, vol. 174, no. 1, pp. 329-336, 2006.

[17] J. J. Liu and M. Yamamoto, "A backward problem for the timefractional diffusion equation," Applicable Analysis, vol. 89, no. 11, pp. 1769-1788, 2010.
[18] W. McLean, "Regularity of solutions to a time-fractional diffusion equation," The ANZIAM Journal, vol. 52, no. 2, pp. 123-138, 2010.

[19] J. Prüss, Evolutionary Integral Equations and Applications, vol. 87, Birkhäuser, Basel, Switzerland, 1993.

[20] A. A. Kilbas, H. M. Srivastava, and J. J. Trujillo, Theory and Applications of Fractional Differential Equations, Elsevier, 2006.

[21] S. G. Samko, A. A. Kilbas, and O. I. Marichev, Fractional Integrals and Drivatives, Gordon and Breach Science, Yverdon, Switzerland, 1993.

[22] D. A. Murio, "Stable numerical solution of a fractional-diffusion inverse heat conduction problem," Computers \& Mathematics with Applications, vol. 53, no. 10, pp. 1492-1501, 2007.

[23] H. W. Engl, M. Hanke, and A. Neubauer, Regularization of Inverse Problems, Kluwer Academic, 1996.

[24] L. Yang, Z.-C. Deng, J.-N. Yu, and G.-W. Luo, “Two regularization strategies for an evolutional type inverse heat source problem," Journal of Physics A, vol. 42, no. 36, Article ID 365203, 16 pages, 2009.

[25] L. Yang, Z.-C. Deng, J.-N. Yu, and G.-W. Luo, “Optimization method for the inverse problem of reconstructing the source term in a parabolic equation," Mathematics and Computers in Simulation, vol. 80, no. 2, pp. 314-326, 2009.

[26] L. Yang, M. Dehghan, J.-N. Yu, and G.-W. Luo, "Inverse problem of time-dependent heat sources numerical reconstruction," Mathematics and Computers in Simulation, vol. 81, no. 8, pp. 1656-1672, 2011.

[27] L. Yang, J. N. Yu, G. W. Luo, and Z. C. Deng, "Reconstruction of a space and time dependent heat source from finite measurement data," International Journal of Heat and Mass Transfer, vol. 55, pp. 6573-6581, 2012.

[28] L. Yang, J. N. Yu, G. W. Luo, and Z. C. Deng, "Numerical identification of source terms for a two dimensional heat conduction problem in polar coordinate system," Applied Mathematical Modelling, vol. 37, pp. 939-957, 2013.

[29] F. F. Dou and Y. C. Hon, "Kernel-based approximation for Cauchy problem of the time-fractional diffusion equation," Engineering Analysis with Boundary Elements, vol. 36, no. 9, pp. 1344-1352, 2012.

[30] Y. C. Hon and T. Wei, "A fundamental solution method for inverse heat conduction problem," Engineering Analysis With Boundary Elements, vol. 28, no. 5, pp. 489-495, 2004.

[31] J. Cheng, J. Nakagawa, M. Yamamoto, and T. Yamazaki, "Uniqueness in an inverse problem for a one-dimensional fractional diffusion equation," Inverse Problems, vol. 25, no. 11, pp. 1-16, 2009.

[32] B. Jin and W. Rundell, "An inverse problem for a onedimensional time-fractional diffusion problem," Inverse Problems, vol. 28, no. 7, Article ID 075010, 19 pages, 2012.

[33] D. N. Hào, "A mollification method for ill-posed problems," Numerische Mathematik, vol. 68, no. 4, pp. 469-506, 1994.

[34] U. Tautenhahn, "Optimality for ill-posed problems under general source conditions," Numerical Functional Analysis and Optimization, vol. 19, no. 3-4, pp. 377-398, 1998. 


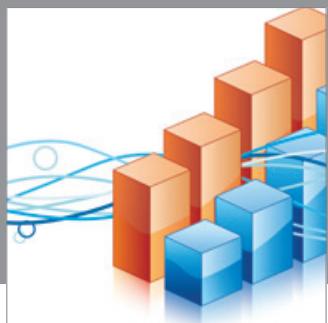

Advances in

Operations Research

mansans

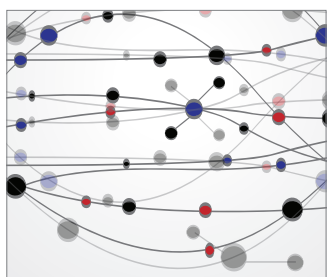

The Scientific World Journal
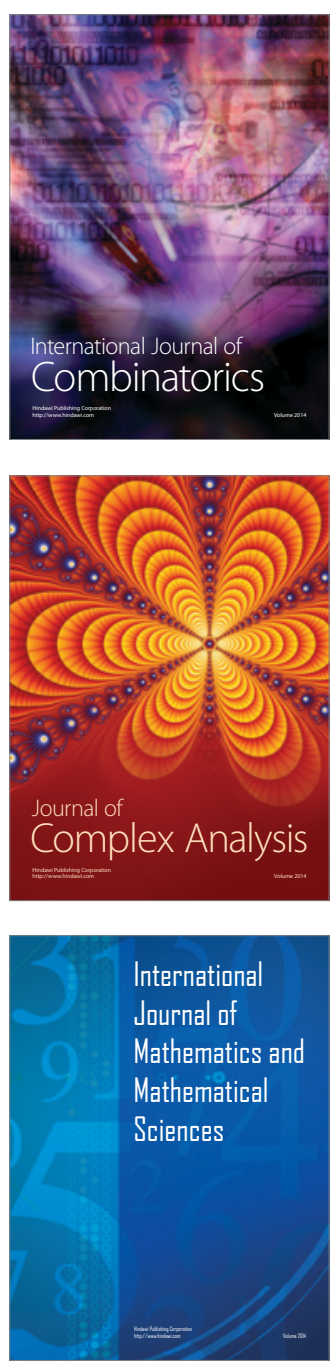
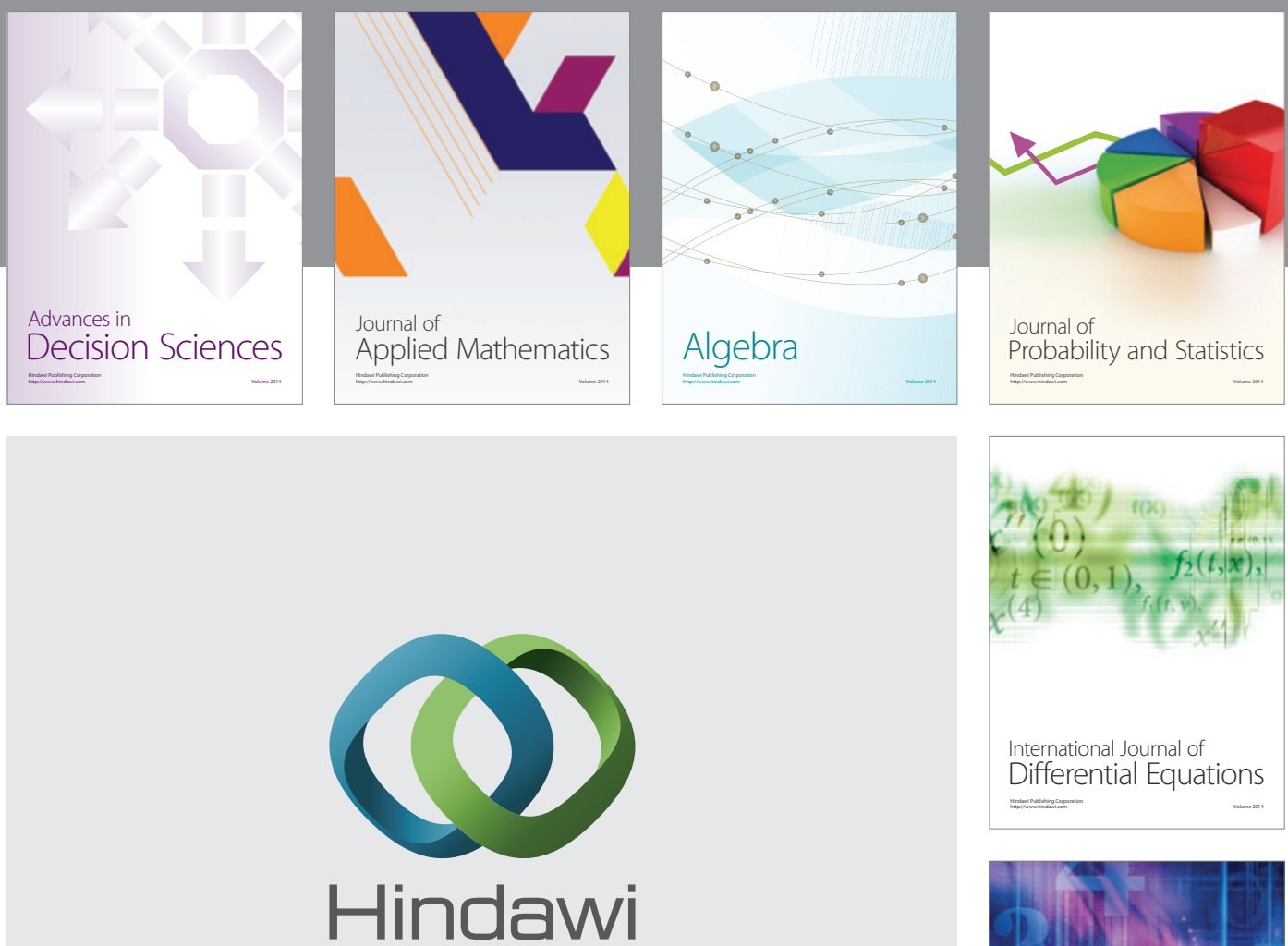

Submit your manuscripts at http://www.hindawi.com
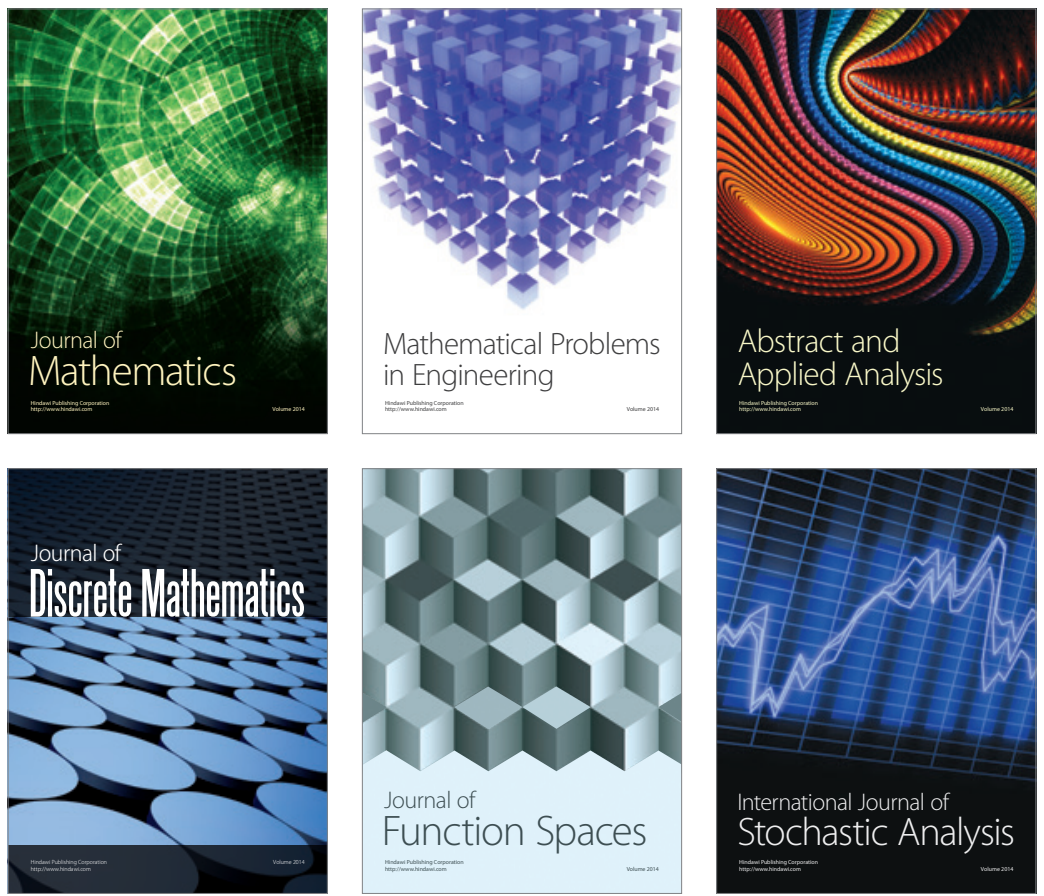

Journal of

Function Spaces

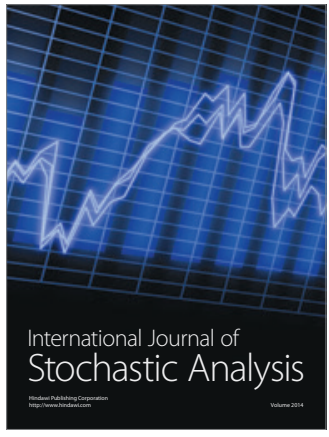

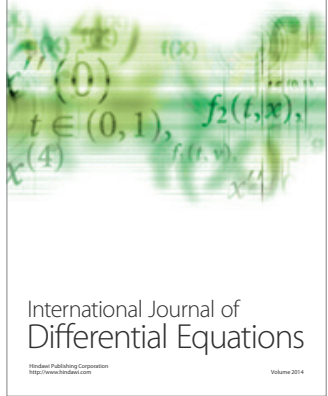
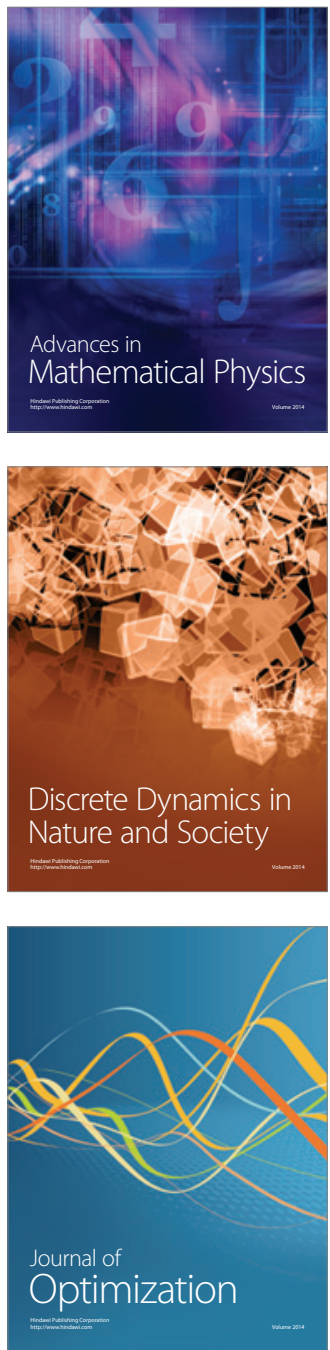